\section{Neue Vertretungsregelungen für Vertragsärzte}

Die Organisation der Vertretung in der Arztpraxis war für den Vertragsarzt schon immer ein schwieriges Feld. Dies gilt unabhängig von der Art der geführten Praxis. Hierbei stehen Berufsausübungsgemeinschaften und Praxisgemeinschaften vor vergleichbaren Problemen wie Medizinische Versorgungszentren. Auf den 1. Blick korrespondieren unübersichtliche Vorgaben in $\S 32$ Abs. 1 Ärzte-ZV mit erheblichen Risiken. $\mathrm{Zu}$ berücksichtigen ist nämlich, dass die Abrechnung im Rahmen der Vertretung Teil der Prüfung der Rechtmäßigkeit und Plausibilität der Abrechnung in der vertragsärztlichen Versorgung gemäß § 106a SGB V ist und somit durch die Kassenärztlichen Vereinigungen eindeutig regressierbar ist (vgl. BSG, Beschl. v. 08.09.2004; Az.: B 6 KA 25/04 B).

Eine besondere Dynamik hat die Vertretungsproblematik durch ein neueres Urteil des Bundessozialgerichts vom 14.12.2011 (Az.: B 6 KA 31/10 R) erhalten. Die Kassenärztlichen Vereinigungen haben bereits infolge dieses Urteils ihre Vorgaben zu den Vertretungsregelungen angepasst bzw. die Verwaltungspraxis geändert.

\section{Die Vertretungsregelung nach $\S$ 32 Abs. 1 Ärzte-ZV}

Schon dem ärztlichen Berufsrecht sind Regelungen zur Vertretung zu entnehmen. Nach $§ 20$ Abs. 1 Satz 2 der MusterBerufsordnung (MBO) ist eine Vertretung nur bei Fachgebietsidentität des Vertreters möglich. Grundlegende Vorgaben für das Vertretungsrecht im vertragsärztlichen Bereich sind in $\S 32$ Abs. 1 Ärzte-ZV enthalten.

Nach dieser Vorschrift hat der Vertragsarzt die vertragsärztliche Tätigkeit persönlich in freier Praxis auszuüben. Dies ist bekannt als die sog. Pflicht zur persönlichen Leistungserbringung. Im Rahmen der Vertretung muss der Vertragsarzt aufgrund dieser Pflicht, insbesondere dem Vertretungsgrund, der Vertretungsdauer und der Person des Vertreters besondere Beachtung schenken.

\section{Vertretung nur in engem Rahmen zulässig}

Vertretungsgründe nach $\S 32$ Abs. 1 S. 1 u. 2 Ärzte-ZV sind Krankheit, Urlaub, Teilnahme an einer ärztlichen Fortbildung, einer Wehrübung oder die Entbindung einer Vertragsärztin. Zu diesen Vertretungsgründen kommen in $\S 32$ Abs. 2 S. 2 Nr. 1-3 Ärzte-ZV noch Vertretungen aus Gründen der Sicherstellung der vertragsärztlichen Versorgung, zu Zeiten der Erziehung von Kindern sowie zur Pflege eines pflegebedürftigen nahen Angehörigen in häuslicher Umgebung hinzu. In diesen Fällen ist allerdings eine Genehmigung durch die Kassenärztliche Vereinigung erforderlich, während für die übrigen Vertretungsgründe nur eine Anzeigepflicht gegenüber der Kassenärztlichen Vereinigung besteht, wenn die in $\S 32$ Abs. 1 Satz 4 Ärzte-ZV genannte Vertretungsdauer „länger als 1 Woche“ dauert.

Die in $\S 32$ Abs. 1 Ärzte-ZV genannten Vertretungsgründe sind abschließend und die Vertretung damit nur innerhalb dieses engumgrenzten Rahmens möglich (vgl. BSG, Urt. v. 14.12.2011; Az.: B 6 KA 31/10 $\mathrm{R} ;$ ).

\section{Vertretungsdauer \\ $\nabla$}

Hinsichtlich der Vertretungsdauer sind unterschiedliche Fristen zu beachten und einzuhalten.

Nach $\S 32$ Abs. 1 S. 2 Ärzte-ZV kann die Vertretung bis zu 3 Monate binnen eines Zeitraums von 12 Monaten dauern, wobei das Kalenderjahr unbeachtet bleibt. Ausschließlich eine Vertragsärztin kann sich bei einer Entbindung bis zu 12 Monate vertreten lassen. Bei Krankheit, längeren Urlauben oder einer Fortbildung wirft dies in der Regel keine Probleme auf.

Problematisch ist allerdings in diesem $\mathrm{Zu}$ sammenhang, dass $\S 32$ Abs. 1 S. 2 ÄrzteZV keine Vorgaben über nur tageweise, aber regelmäßig durchgeführte Vertretungen enthält. Die Rechtsprechung hat in diesem Zusammenhang entschieden, dass hierbei keine Addition der einzelnen

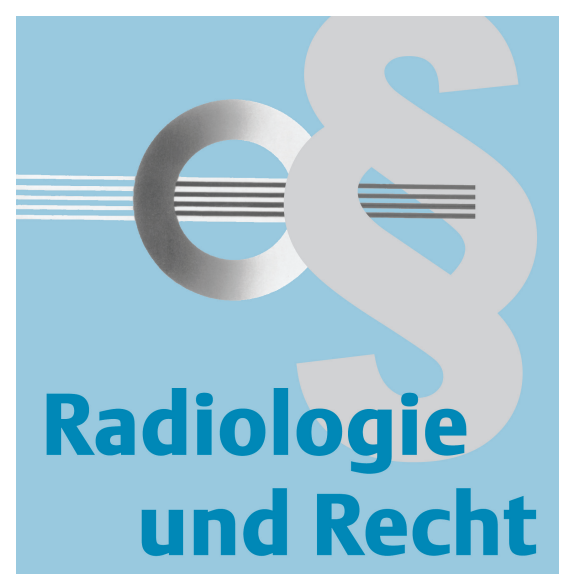

Vertretertage erfolgt, sondern der Zeitraum mit der 1 . Vertretung beginnt, wobei eine höchstrichterliche Entscheidung noch aussteht (SG Mainz, Urt. v. 24.02.1988; Az.: S 1 a Ka 76/87). In der Konsequenz heißt dies, dass bei Vertretungen z. B. an 2 Nachmittagen in der Woche, nach 3 Monaten die gesamte Vertretungszeit „aufgebraucht“ ist. Eine andere Beurteilung ergibt sich aufgrund eines Urteils des SG Marburg vom 08.12.2010 (Az.: S 12 KA 30/10) nur bei rein sporadischen Vertretungen. Das Gericht führt aus, „dass ein Vertretungsfall nur dann angenommen werden kann, wenn der Vertragsarzt aus einem besonderen Grund an der Ausübung seiner Praxis verhindert sei, d.h. nicht nur stundenweise abwesend ist und die Praxis geschlossen bleibt“.

Längere Vertretungszeiten ergeben sich nur in den besonderen Fällen des $\S 32$ Abs. 2 S. 2 Nr. 2 bis 3 Ärzte-ZV. Ein Vertreter kann während der Zeiten der Erziehung von Kindern bis zu einer Dauer von 36 Monaten beschäftigt werden, wobei dieser Zeitraum nicht zusammenhängend genommen werden muss sowie während der Pflege eines pflegebedürftigen nahen Angehörigen in häuslicher Umgebung bis zu einer Dauer von 6 Monaten.

Schließlich sollte beachtet werden, dass bei einer Vertretungsdauer, die 1 Woche dauert, diese der Kassenärztlichen Vereinigung mitzuteilen ist. Bei einer Überschreitung der Vertretungsdauer von 1 Monat, innerhalb von 12 Monaten, ist die Kassenärztliche Vereinigung berechtigt, zu prüfen, ob der Vertreter die entsprechenden Vertretungsvoraussetzungen erfüllt. 


\section{Anforderungen an den Vertreter $\nabla$}

Der Vertreter selbst muss nach $\S 32$ Abs. 1 S. 4 Ärzte-ZV Vertragsarzt sein oder die Bedingungen erfüllen, um in das Arztregister nach $\S 3$ Abs. 2 Ärzte-ZV eingetragen zu werden. Gemäß $\S 3$ Abs. 2 Ärzte-ZV sind die Voraussetzungen für die Eintragung die Approbation als Arzt und der erfolgreiche Abschluss einer allgemeinmedizinischen Weiterbildung oder einer Weiterbildung in einem anderen Fachgebiet mit der Befugnis zum Führen einer entsprechenden Gebietsbezeichnung bzw. der Nachweis einer Qualifikation, die gemäß § 95a Abs. 4 und 5 SGB V anerkannt ist. Darüber hinaus können ärztliche Leistungen, deren Abrechenbarkeit den Nachweis einer besonderen Qualifikation erfordert, von einem Vertragsarzt im Fall seiner Vertretung nur dann abgerechnet werden, wenn auch der Vertreter die erforderliche Qualifikation besitzt (vgl. BSG, Urt. v. 28.01.1998, Az.: B 6 KA 93/96 R). Wird daher z. B. ein Facharzt für Radiologie mit einer Abrechnungsgenehmigung nach der Kernspintomografie-Vereinbarung nach $\S 135$ Abs. 2 SGB V vertreten, sind die MRT-Leistungen in dieser Zeit weiterhin nur abrechnungsfähig, wenn auch der Vertreter über die Abrechnungsgenehmigung nach der Kernspintomografie-Vereinbarung verfügt. Der vertretene Arzt hat dabei nach $\S 14$ Abs. 1 des Bundesmantelvertrags-Ärzte (BMV-Ä) die Pflicht, dies zu überprüfen.

Die bereits im Berufsrecht enthaltene Forderung nach einer Fachidentität zwischen Vertreter und Vertretenem wird im Vertragsarztrecht auf die Versorgungsbereiche erweitert. Bedeutsam sind insoweit die Ausführungen des BSG in seinem Urteil vom 14.12.2011 (Az.: B 6 KA 31/10 R). Das BSG hatte in dieser Entscheidung über die sachlich-rechnerische Richtigstellung der Abrechnungen einer Gemeinschaftspraxis zu entscheiden, in der ein Facharzt für Innere Medizin mit dem Schwerpunkt Gastroenterologie, der an der fachärztlichen Versorgung teilnahm und ein hausärztlich tätiger Internist ohne Schwerpunktbezeichnung zusammen praktizierten. Dabei wurden im Rahmen einer praxisinternen Vertretung vom hausärztlichen Internisten fachärztliche Leistungen erbracht, was von der Kassenärztlichen Vereinigung beanstandet wurde. Das BSG stellte fest, dass Leistungen der haus- bzw. der fachärztlichen Versorgung nach $\S 73$ SGB V nur von den jeweils daran teilnehmenden Ärzten erbracht werden dürften.
Auch wenn vertragsärztliche Leistungen der Gemeinschaftspraxis und nicht einzelnen Ärzten zugeordnet würden, bedeute dies nicht, dass die beteiligten Ärzte Fachgebietsgrenzen, Qualifikationsvorgaben und sonstige für die Abrechnung wichtige Vorgaben umgehen dürften.

\section{Keine gegenseitige Vertretung von Ärzten in BAG und MVZ $\nabla$}

Dies gelte auch im Rahmen der Vertretung. Das BSG stellt zudem klar, dass Behandlungen durch einen Gemeinschaftspraxispartner keine Vertretungen im Sinne des $\S 32$ Abs. 1 Ärzte-ZV darstellen, da die Gemeinschaftspraxis (= Berufsausübungsgemeinschaft) nach außen wie ein einziger Arzt auftrete. Eine Vertretung liege erst dann vor, wenn der Ausfall eines Praxispartners nicht durch die verbliebenen Ärzte aufgefangen werden könne und ein externer Arzt zur Leistungserbringung herangezogen werden müsse. Die Grundsätze des Urteils des BSG sind auf MVZ entsprechend anwendbar.

\section{Umsetzung der Vorgaben durch die Kassenärztlichen Vereinigun- gen $\nabla$}

Die Kassenärztlichen Vereinigungen planen ihre Vertretungsregelungen oder ihre Verwaltungspraxis an die Grundsätze dieses Urteils des BSG vom 14.12.2011 (Az.: B 6 KA 31/10 R) anzupassen oder haben dies bereits getan.

Im Bereich der Kassenärztlichen Vereinigung Westfalen-Lippe (KVWL) gilt z.B. seit dem 01.01.2014 eine neue Vertretungsregelung, die die Anforderungen des BSG-Urteils aufnimmt (vgl. praxisintern, Nr. 8 vom 27. August 2013). Die KVWL führt dabei 2 neue Begrifflichkeiten ein: Die sog. externe, echte Vertretung und die sog. interne, unechte Vertretung. Eine „echte Vertretung“ bedeutet, dass ein Vertragsarzt durch einen externen Vertreter vertreten wird. Vertragsärzte, die im Rahmen einer Berufsausübungsgemeinschaft als Gesellschafter oder angestellte Ärzte tätig sind, können daher keine externen Vertreter sein.

Eine „unechte Vertretung“ stellt demgegenüber im eigentlichen Sinn keine Vertretung im Sinne des $\S 32$ Abs. 1 Ärzte-ZV dar, sondern beschreibt den Fall, dass innerhalb einer BAG oder eines MVZ ein
Vertragsarzt die Vertretung eines abwesenden Praxiskollegen übernimmt. Rechtlich sei dies, wie im obigen Urteil ausgeführt, nur die Behandlung durch einen anderen Arzt der BAG bzw. des MVZ, aber keine Vertretung. Die ärztlichen Leistungen des Praxiskollegen sind daher nicht auf Vertretungsschein möglich.

Hinsichtlich der Qualifikationserfordernisse hat die KVWL das BSG-Urteil bereits umgesetzt. Sowohl bei der echten wie auch bei der unechten Vertretung müssen die entsprechenden Qualifikationen vorliegen. Der Nachweis erfolgt bei Vertragsärzten über die entsprechende qualifikationsgebundene Abrechnungsgenehmigung, bei Nicht-Vertragsärzten über ein von der Kassenärztlichen Vereinigung ausgestelltes sogenanntes Qualifkationstestat.

Hinsichtlich des Abrechnungsverfahrens stellt die KVWL folgende Regelung auf: Bei einer „echten“ Vertretung innerhalb der Praxis des Vertretenen werden danach die Leistungen unter dessen LANR abgerechnet. Dies gilt unabhängig davon, ob es sich bei dem Vertreter um einen vertragsärztlich tätigen Arzt handelt oder nicht. Bei einer Vertretung in der Praxis des Vertreters werden die Leistungen auf dessen LANR auf einem Vertretungsschein abgerechnet.

Bei der „unechten“Vertretung müssen die Leistungen, die für einen abwesenden Praxispartner in einer BAG oder einem MVZ erbracht werden, über die eigene LANR des Praxiskollegen gekennzeichnet und abgerechnet werden. Dies gilt auch für fachgleiche BAG und die Vertretung fachgleicher Kollegen in einem MVZ.

\section{Vertretungen in der Praxisge- meinschaft 7}

Vertretungen können in engen Grenzen auch in einer Praxisgemeinschaft vorkommen, wobei der Grad der Patientenidentität hier die größte Gefahr beinhaltet. Bis zu einem Grad von 20\%, bezogen auf die gesamte Praxisgemeinschaft, wird eine solche zwar von den Kassenärztlichen Vereinigungen nicht direkt sanktioniert und unter Umständen durch hausinterne Regelungen auch bis zu 30\% geduldet. Allerdings bestehen keine Rechtsansprüche auf eine derartige hausinterne Quotenbildung, sodass Ärzte einer Praxisgemeinschaft gut beraten sind, keine $\mathrm{Pa}$ - 
tientenidentität von mehr als 20\% aufzuweisen.

Problematisch sind daher gegenseitige Vertretungen in einer Praxisgemeinschaft, wenn diese zu einer erhöhten Patientenidentität führen. Im Verlauf der Überprüfung werden die in Vertretung erbrachten Fälle analysiert und festgestellt, ob es „echte“"Vertretungsfälle im Sinne einer Notfallbehandlung sind. Routinemäßige Behandlungen müssen von der Praxisgemeinschaft im Vorfeld derart geleitet werden, dass diese während der Abwesenheitszeit nicht anfallen. Büronachmittage oder externe Verpflichtungen, die zu standardisierten Vertretungen führen, können in einer Praxisgemeinschaft ebenfalls nicht vorgenommen werden. Besonders problematisch gestaltet sich die Situation, wenn die in der Praxisgemeinschaft verbundenen Ärzte einen weiteren Arzt, jeweils hälftig anstellen und über diesen wechselseitige Vertretungen vornehmen. Aufgrund der ebenfalls vorhandenen Zugehörigkeit zu der anderen Praxis ist während der Anwesenheitszeiten des betreffenden Vertragsarztes eine „echte“ Vertretung durch den angestellten Arztes, egal in welcher Praxis, nicht möglich.

\section{Fazit}

$\nabla$

Durch das BSG-Urteil und der hieraus resultierenden Neuregelung der Praxisvertretungen wird die Möglichkeit echter Vertretungen nach $\S 32$ Abs. 1 Ärzte-ZV erheblich eingeschränkt. Dies erfolgt zwar nicht im Sinne der Vertragsärzte, verschafft aber zumindest Rechtssicherheit. Besonders wichtig ist, dass Vertragsärzte vor Bestellung eines Vertreters unbedingt überprüfen, ob dieser die entsprechenden Qualifikationsnachweise zur Erbringung und Abrechnung der Leistungen besitzt.

Allerdings eröffnen sich auch neue Probleme durch die veränderten Vertretungsregelungen. Es wurde z.B. nicht berücksichtigt, wie Vertretungsleistungen durch Ärzte, die in einer Berufsausübungsgemeinschaft oder einem MVZ tätig sind und nur über einen hälftigen Versorgungsauftrag nach $§ 95$ Abs. 3 Satz 1 SGB V i.V.m. § 19a Abs. 2 Ärzte-ZV verfügen, eingeordnet werden. Für eine „unechte“ Vertretung spricht, dass die Tätigkeit räumlich in der Praxis vorgenommen wird, in dem der Arzt auch sonst tätig ist. Zudem ist er mit der Vertretung eines Praxispartners befasst. Auf der anderen Seite ist ein Arzt mit einem nur hälftigen Versorgungsauftrag im Rahmen der Vertretungstätigkeit außerhalb seines unmittelbaren vertragsärztlichen Versorgungsauftrages nach § 19a Abs. 2 Ärzte-ZV tätig. Aus dieser Unsicherheit können erhebliche Risiken bezüglich der Frage erwachsen, wer die Leistungen abrechnen muss, bis hin zu einer Plausibilitätsprüfung mit ungewissem Ergebnis, sowohl hinsichtlich der Vertretungstätigkeit als auch hinsichtlich der Überschreitung der Tagesund Quartalsprofile des einzelnen Arztes.

Es bleibt daher abzuwarten, wie in der Praxis mit diesen Fällen umzugehen sein wird. Es ist anzuraten, bei den zuständigen Kassenärztlichen Vereinigungen zu genauen Regelungen zu fragen und bis dahin erhebliche Vorsicht bei der Vertretung walten zu lassen.

\section{Lic. iur. can. Urs Fabian Frigger Rechtsanwalt}

\section{Jens Remmert, LL.M.}

Rechtsanwalt

Rechtsanwälte Wigge

Scharnhorststr. 40

48151 Münster

Telefon: (0251) 53595-0

Telefax: (0251) 53595-99

E-Mail: kanzlei@ra-wigge.de

www.ra-wigge.de 